\title{
Improved Modified Chaotic Invasive Weed Optimization Approach to Solve Multi-Target Assignment for Humanoid Robot
}

\author{
Abhishek Kumar Kashyap ${ }^{1 *}$, Dayal Parhi ${ }^{2}$, Anish Pandey ${ }^{3}$ \\ ${ }^{1,2}$ Mechanical Engineering Department, National Institute of Technology, Rourkela-769008, Odisha, India \\ ${ }^{3}$ School of Mechanical Engineering, Kalinga Institute of Industrial Technology, Bhubaneswar-751024, Odisha, India \\ Email: ${ }^{1}$ techmech46@gmail.com \\ *Corresponding Author
}

\begin{abstract}
The paper presents an improved modified chaotic invasive weed optimization (IMCIWO) approach for solving a multi-target assignment for humanoid robot navigation. MCIWO is improved by utilizing the Bezier curve for smoothing the path and replaces the conventional split lines. In order to efficiently determine subsequent locations of the robot from the present location on the provided terrain, such that the routes to be specifically generated for the robot are relatively small, with the shortest distance from the barriers that have been generated using the IMCIWO approach. The MCIWO approach designed the path based on obstacles and targets position which is further smoothened by the Bezier curve. Simulations are performed which is further validated by real-time experiments in WEBOT and NAO robot respectively. They show good effectiveness with each other with a deviation of under $5 \%$. Ultimately, the superiority of the developed approach is examined with existing techniques for navigation, and findings are substantially improved.
\end{abstract}

Keywords-Humanoid robot, Multiple target problem, Bezier curve, Modified Chaotic Invasive Weed Optimization

\section{INTRODUCTION}

In the analysis of collective behavior and the evolving characteristics of complicated structures with a specific social structure, Swarm Intelligence introduced a significant aspect of artificial intelligence. This article will address this subject. The strategies for navigation and trajectory mapping are mainly graded as local or sensor-oriented techniques and global or model-based techniques. The robot is uncertain of terrain and arranges instant identification and preparation to avoid barriers in sensor-based techniques. The robot knows the local environments with barrier locations in model-based techniques and so designs the route by planning the terrain from the outset. In the past decades, studies in humanoid navigation and robotics have been the subject of many scientists. Here several of them are described.

Various nature-inspired approach [1]-[6] has been employed in various types of robots to date. In order to build a methodology in real-time strategy for humanoids pondering point by point height acquaintance, Karkowski et al. [7] have utilized the $\mathrm{A} *$ method and dynamic $3 \mathrm{D}$ motion. Ido et al. [8] have utilized movement capture data to submit a pattern on a view basis and analyzed humanoid gait patterns.
Kashyap et al. [9] have describes the dynamics of the robot based on whole-body control. In addition, they have simplified the study of complete structure using the 3D-LIPM model [10]. In order to balance an accurate multi-structural line, Zhong and Ai [11] have altered the simple Ant colony method. A minimum task gap has been observed between workstations. Habib et al. [12] have employed the integrated Voronoi diagram method and updated ACO in a step by step motion designing for robots. Kashyap et al. [13] accomplished a secure path for the biped robot using hybrid control techniques. Kumar et al. [14], [15] have applied various techniques for shunning obstacles and achieving the target in the humanoid robot. Rath et al. [16] have developed and implemented a fuzzy-based technique in humanoid robots to map a better path. Chinmaya et al. [17] have modified the path for a humanoid robot in an obscure environment and verified its performance using various simulations and experiments.

To fit PID controller specifications for the hydraulic turbine system, Navid and Mohsen [18] have established an invasive weed optimization approach. In addition, in electronic implementations, Karimkashi and Kishk [19] have also employed the IWO approach. In addition, Naidu and Ojha [20] have described the latest hybrid IWO approach and contrasted the findings for a quadratic approximate dilemma with a regular genetic algorithm. A modified invasive weed optimization (MIWO) approach has been used by abedinia et al. [21] to adjust the specifications of the fuzzy PID controller in a multi-objective power framework. Compared to other methods it has been found that the proposed algorithm operated efficiently.

Studying the available papers suggests that the robots are guided towards the target but mostly focusses on mobile robots rather than humanoid robots. The papers available for humanoids robots focus on stabilization primarily. Precisely, navigations of a humanoid robot in a terrain consisting of multiple targets is an untouched topic, therefore, this study focusses on the same. MCIWO technique is implemented to take obstacles and targets position as input which provides an initial turning angle for navigation. Sharp turn can leads to reduce the balance of a humanoid robot or increase the path 
length and time spent. Therefore, to optimize these parameters and provide better stabilization, the Bezier curve is implemented in MCIWO to improve its property and provide smooth turning.

The major breakthrough of this paper is solving the multitarget assignment for a humanoid robot using the IMCIWO approach. The rest portion of the paper is prepared as follows. The architecture of the proposed approach is portrayed in Section II, followed by its implementation in simulation and experimental terrain in Section III. A comparison with the existing controller is performed in Section IV and ultimately, the conclusions are described in Section V.

\section{ARCHITECHTURE OF PROPOSED APPROACH}

This section describes the framework of the MCIWO approach and its improvement using the Bezier curve.

\section{A. Modified chaotic invasive weed optimization approach}

Mehrabain and Lucas [22] have proposed a stochastic computational optimization tool IWO approach in 2006, which is inspired by the colonization of the weeds. Since employing the evacuation concepts, weeds are used in the field and absorbed in the opening areas inside the crop. Unused capital in the plant and creates fresh seeds that allow the budding of the weeds. These seeds are arbitrarily scattered in a region and develop into a new flora weed. The overall amount of weeds in the system has trouble for the entity with other weeds because those weeds are stronger and more seeds can thrive.

For IWO algorithms, the necessary procedures are:

- Select the number of problematic variables and allocate the range of values to each variable. A certain amount of seeds is unevenly scattered in an $\mathrm{N}$-dimensional system and each seed holds an altered location, that is to say, a preliminary approach.

- Depending on the use of unused land property, a seedlings plant is generated in each preliminary seed and additional seeds are generated at the fitness of every plant. The amount of seeds generated from each plant has been determined with the following formula in the reproductive method. It allows to make a contribution to the forthcoming fertilization process by means of all weeds produced. This is beneficial because the weakest fitness quality of the weed also offers several valuable knowledge for engaging in the developmental mechanism under some conditions.

$$
P=\text { Floor }\left[P^{\min }+\frac{\left(f i t-f i t^{\min }\right)^{n}}{\left(f i t^{\max }-f i t^{\min }\right)^{n}} * P^{\max }\right]
$$

where $\left[P^{\min }, P^{\max }\right]$ and $\left[f i t^{\min }, f i t^{\max }\right]$ are the range of production of each plant and fitness value in the colony.

$$
Y^{k+1}=\cos \left(k \cos ^{-1}\left(Y^{k}\right)\right)
$$

In addition, the standard deviation factor $\left(\sigma^{g e n}\right)$ utilized to describe the seed location is provided as follows:

$$
\sigma^{g e n}=\frac{\left(g^{\max }-\text { gen }\right)^{n}}{\left(g^{\max }\right)^{n}} * \mid \cos (\text { gen }) \mid *\left(\sigma^{i}-\sigma^{f}\right)+\sigma^{f}
$$

Where $g e n^{\max }$ the highest generation count, $n$ is the integer value of the modulated signal and i.e. $\left(\sigma^{i}-\sigma^{f}\right)$ is the range of the standard deviation. Not only does the terminology $\mid \cos ($ gen $) \mid$ help to detect the global optimal response, it also increases the evaluation area through the use of minimal resources. The system conducts more searches than the regular IWO technique after modifications. The fitness of these new seeds is also assessed and ranks mostly with parent plants when the reproductive systems have been developed. It should be noted that the permissible quantity of plants should not be greater than $P^{\max }$. The lower classification plants then are exempt from competition. This method continues until you have reached the full amount of iterations. The flowchart MCIWO is described in Fig. 1. The process initializes and the robot senses the location of obstacles and targets presents in the terrain. The possible paths are designed based on their respective fitness values. The location and velocity of the robot are updated and the best path is selected which has the highest fitness and lower fitness paths are eliminated. The process repeats until the robot reaches the target and optimality is obtained.

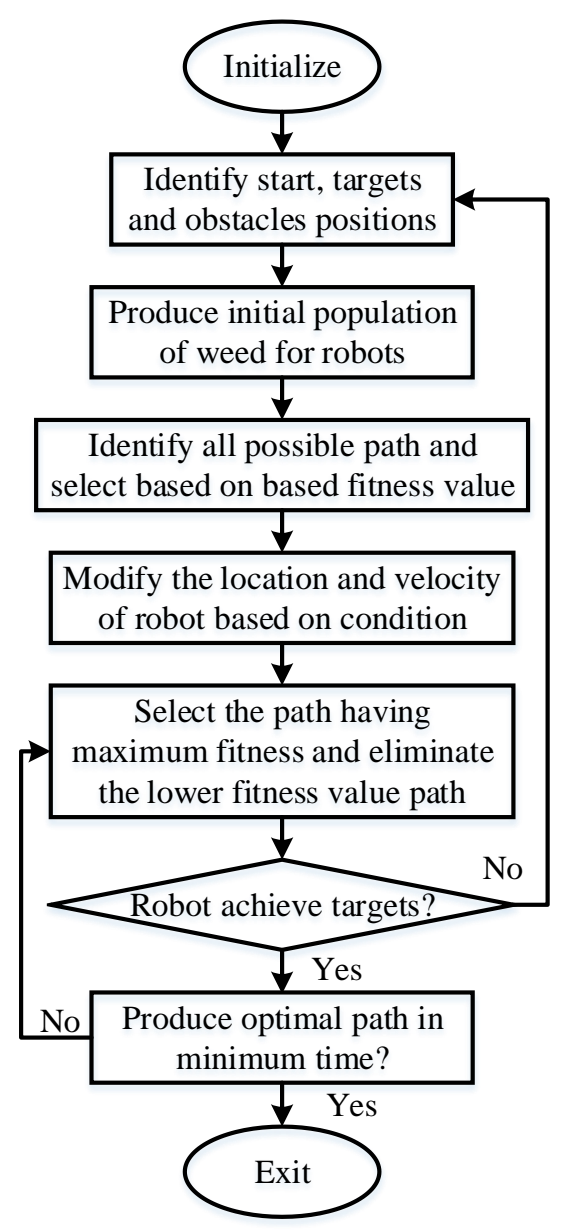

Fig. 1. Flowchart of MCIWO for humanoid robot navigation 


\section{B. Bezier curve}

The path has been designed with obstacle avoidance. The turning angle around the obstacle is sharp which can cause the problem in the stabilization of humanoid robots. In order to smoothen the path, the Bezier curve is implemented, which is described in this subsection. In 1962 Pierre Bezier first implemented the Bezier curve for car design [23]. Unlike many other curves such as polynomials or cubic splines, the Bezier curve is located entirely inside the Euclidean space of the Bezier curve command posts. The Bezier curve can be interpreted and rotated by the action of command posts. Bezier curve has less pivotal moments, the points where the curve slope alters its symbol, so it is softer than cubic splines and differs smoother in view of its continuously higher-order variants, through starting position to the goal The Bezier curve travels via the beginning and ending checkpoint, not through the median ones specifying the begin and finish of the curve.

A set of $z+1$ control points $C^{0}, C^{1}, C^{2}, \ldots, C^{z}$, the equivalent Bezier curve $B^{c}$ is presented as:

$$
C(t)=\sum_{j=0}^{z} C^{j} B_{z}^{j}(t), \quad t \in[0,1]
$$

where $B_{z}^{j}(t)$ is represented as Bernstein polynomial which is presented as:

$$
B_{z}^{j}(t)=\left(\begin{array}{c}
z \\
j
\end{array}\right) t^{j}(1-t)^{z-j}, j=0,1,2, \ldots, z
$$

The curve $B^{c}$ is represented in two dimensions as:

$$
\left.\begin{array}{l}
C^{B^{c}}(x, t)=\sum_{j}^{z} C^{j}(x) B_{z}^{j}(t) \\
C^{B^{c}}(y, t)=\sum_{j}^{z} C^{j}(y) B_{z}^{j}(t)
\end{array}\right\}
$$

The length of Bezier curve $B^{c}$ is obtained as:

$$
\begin{aligned}
& l\left(B^{c}\right)=\sum_{i=2}^{n} \sqrt{u+v}, \quad(n=1000) \\
& \left.\begin{array}{l}
u=\left(C^{B^{c}}\left(x, \frac{i}{n}\right)-C^{B^{c}}\left(x, \frac{i-1}{n}\right)\right) \\
v=\left(C^{B^{c}}\left(y, \frac{i}{n}\right)-C^{B^{c}}\left(y, \frac{i-1}{n}\right)\right)
\end{array}\right\}
\end{aligned}
$$

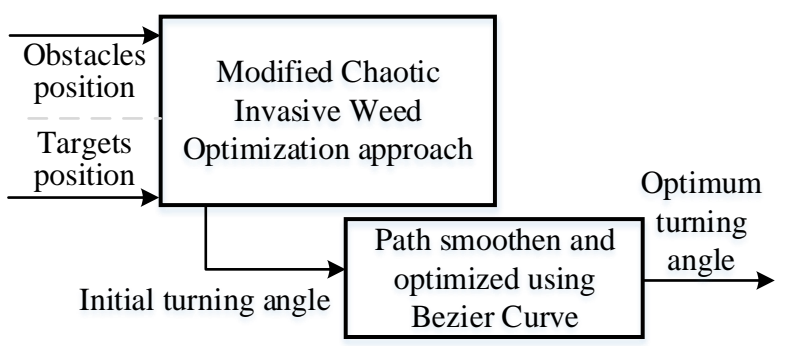

Fig. 2. Procedure of improvement MCIWO approach using Bezier curve to obtain optimum turning angle
The MCIWO approach is improved by implanting the Bezier curve as described in Fig. 2. The obstacles and targets position are implemented in the MCIWO approach which provides an initial turning angle. The robot starts walking and takes a turn when interacted with any obstacles. It provides a sharp turn. It is smoothened by implementing the Bezier curve using the process shown in Fig. 3. It produces some imaginary points between consecutive lines and smoothens the curve. The start and endpoints are taken as $C^{0}$ and $C^{3}$, and $C^{1}$ and $C^{2}$ are the intermediate points. Points, i.e. $C_{1}^{0}, C_{1}^{1}$ and $C_{1}^{2}$ are taken on the line segments. Further, points $C_{2}^{0}, C_{3}^{0}$ and $C_{2}^{1}$ are taken to smoothen it. These lines are connected imaginary and produce a smooth trajectory for a humanoid robot. The approach, IWO, MCIWO, and IMCIWO are compared based on the convergence of optimum travel length against the number of iterations. The graph is described in Fig. 4 shows the IMCIWO approach which is an improved version of the MCIWO approach using the Bezier curve provides optimum path length in contrast to IWO and MCIWO approach. This shows the robustness of the proposed controller. Based on the result obtained, further examinations are carried out with only the IMCIWO approach. Simulations and experiments are performed to examine its efficiency and acceptance is examined by comparing it with the existing controller.

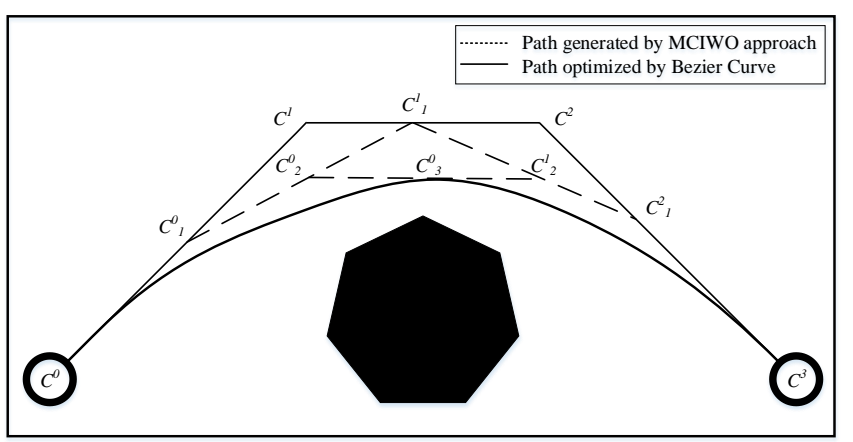

Fig. 3. Improvement of path trajectory using Bezier curve

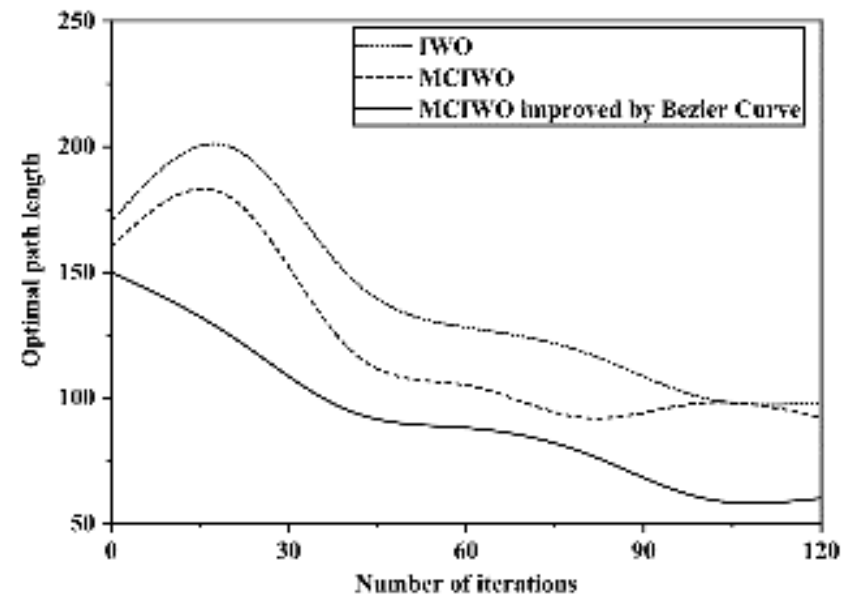

Fig. 4. Comparison of convergence of optimal path length of IWO approach, MCIWO approach and IMCIWO approach 


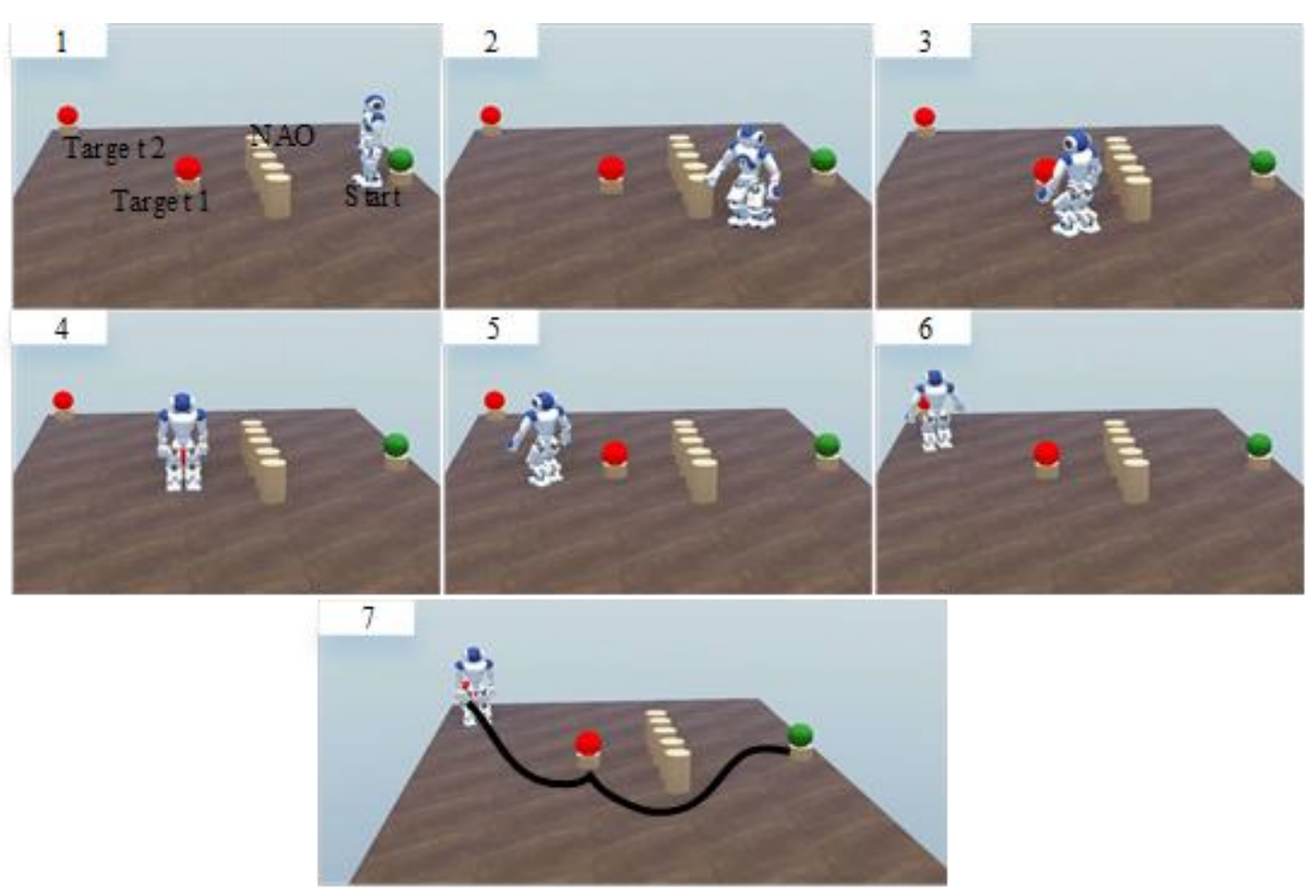

Fig. 5. Simulation snapshot for implementation of IMCIWO approach in multi-target assignment

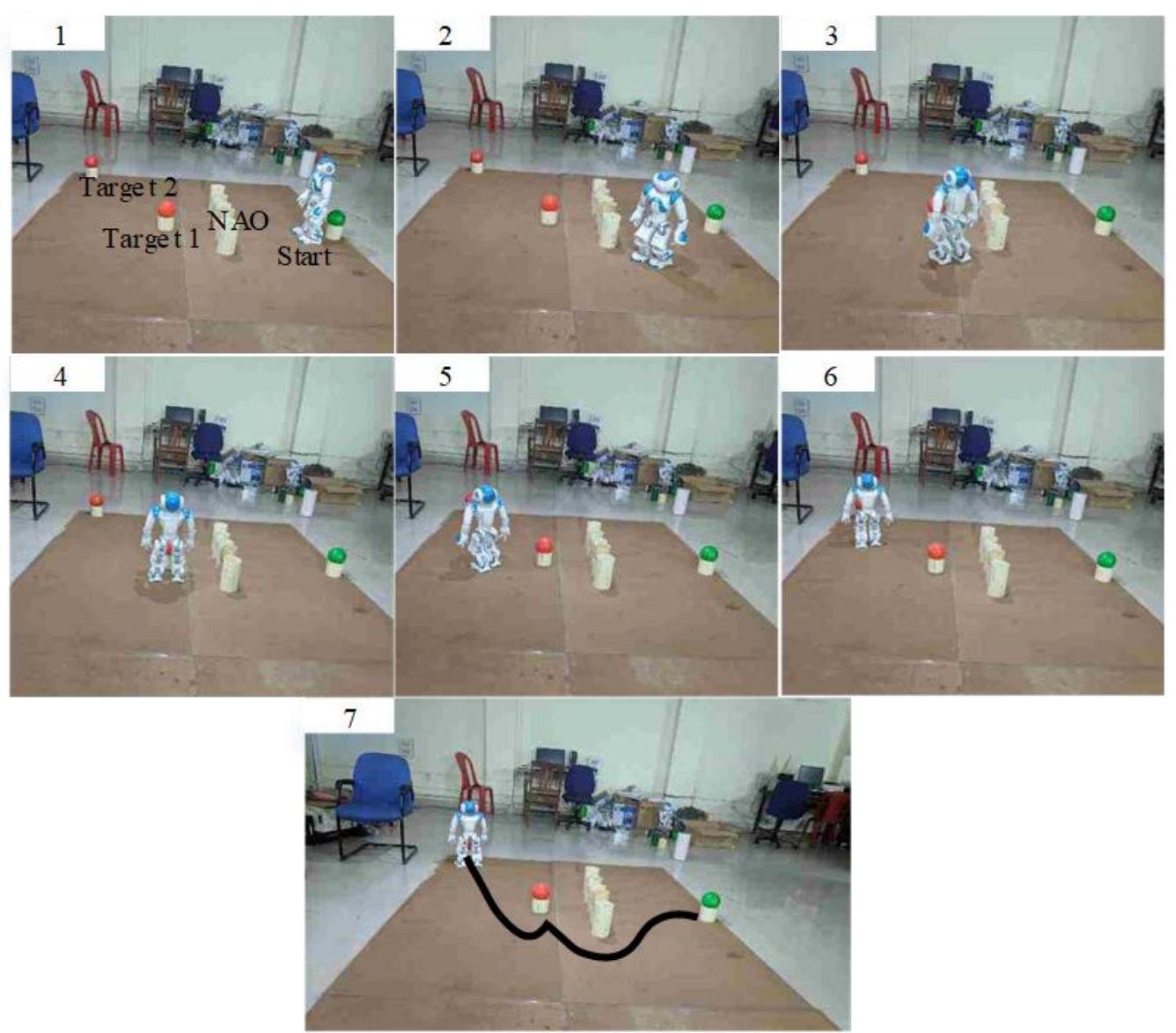

Fig. 6. Experimental snapshot for implementation of IMCIWO approach in multi-target assignment 


\section{IMPLEMENTATION OF PROPOSED APPROACH IN MulTi-TARGET ASSIGNMENT IN SIMLATION AND EXPERIMENTAL TERRAIN}

The robustness of all three approaches (IWO, MCIWO, and IMCIWO) is analyzed and it shows, the IMCIWO approach provides the optimum result. Therefore, in this section, the effectiveness has been analyzed by implementing it in simulated NAO for solving multi-targets assignment. Fig. 5 portrays the snapshots of simulation in WEBOT (3D simulator) that has been carried out by implementing the IMCIWO approach. It shows that the robot is avoiding obstacles and achieving both targets with the optimum path. This result is ratified using the implementation of the IMCIWO approach in experimental terrain in a similar design of terrain and the snapshots are displayed in Fig. 6. The navigational parameters (path length and time spent) for simulations and experiments are necessary to measure so that these can be compared to prove its efficiency. These can be measured with various techniques. The path length and time spent are calculated directly from the WEBOT interface in simulation and used various tools (stopwatch and measuring tape) to calculate for the experiment. These are compared and portrayed in tabular for in Table I and Table II for path length and time spent, respectively. The deviation is recorded under $5 \%$ which shows the results are acceptable and proves the efficiency of the proposed approach.

TABLE I. COMPARISON OF PATH LENGTH IN SIMULATION AND EXPERIMENTAL TERRAINS

\begin{tabular}{|c|c|c|c|}
\hline \multirow{2}{*}{ Sl. No. } & \multicolumn{3}{|c|}{ Path length $(\mathbf{c m})$ using IMCIWO approach } \\
\cline { 2 - 4 } & Simulation & Experiment & Deviation (\%) \\
\hline 1. & 320.3 & 336.7 & 4.87 \\
\hline 2. & 322.6 & 335.1 & 3.73 \\
\hline 3. & 315.7 & 329.2 & 4.1 \\
\hline 4. & 336.1 & 342.1 & 1.75 \\
\hline 5. & 320.1 & 333.6 & 4.05 \\
\hline Avg. & $\mathbf{3 2 2 . 9 6}$ & $\mathbf{3 3 5 . 3 4}$ & $\mathbf{3 . 7}$ \\
\hline
\end{tabular}

TABLE II. COMPARISON OF TIME SPENT IN SIMULATION AND EXPERIMENTAL TERRAINS

\begin{tabular}{|c|c|c|c|}
\hline \multirow{2}{*}{ Sl. No. } & \multicolumn{3}{|c|}{ Time spent (s) using IMCIWO approach } \\
\cline { 2 - 4 } & Simulation & Experiment & Deviation (\%) \\
\hline 1. & 80.34 & 83.16 & 3.39 \\
\hline 2. & 82.54 & 85.01 & 2.91 \\
\hline 3. & 79.21 & 82.91 & 4.46 \\
\hline 4. & 86.37 & 90.12 & 4.16 \\
\hline 5. & 79.01 & 82.59 & 4.33 \\
\hline Avg. & $\mathbf{8 1 . 4 9}$ & $\mathbf{8 4 . 7 6}$ & $\mathbf{3 . 8 5}$ \\
\hline
\end{tabular}

\section{COMPARISON WITH THE EXISTING CONTROLLER}

The robustness and efficiency of the proposed approach are proved. In this section, its acceptance over other controllers is examined. For evaluation, a hybrid artificial bee colony and evolutionary programming (ABC-EP) controller is preferred which has been used by Contreras-Cruz et al. [24] for navigation, which is shown in Fig. 7. Similar terrain has been designed and special care while placing the obstacles, start and target is precisely taken to replicate the preferred article terrain. The robot feed with the IMCIWO approach is guided towards the target. And, based on Fig. 8, it is observed that the path adopted by the IMCIWO approach is smoother and guided the robot closer to the obstacle. This provides an optimum path length for the robot to reach the target. The path length has been compared and described in Table III. The average improvement observed from it is $9.75 \%$, which proves it is acceptable over another navigational controller.

\section{CONCLUSION}

The humanoid robot navigation is a favorite topic for many researchers. A smooth trajectory to avoid collision with obstacles is very important to provide better stability. It is also necessary for obtaining optimized path length and time spent (navigational parameters). To obtain these desired outcomes, the MCIWO approach which is improved by the Bezier curve is utilized. The developed approach works on the two-step mechanism. Initially, the path is plotted by the MCIWO approach by taking the obstacles position and targets position as input.

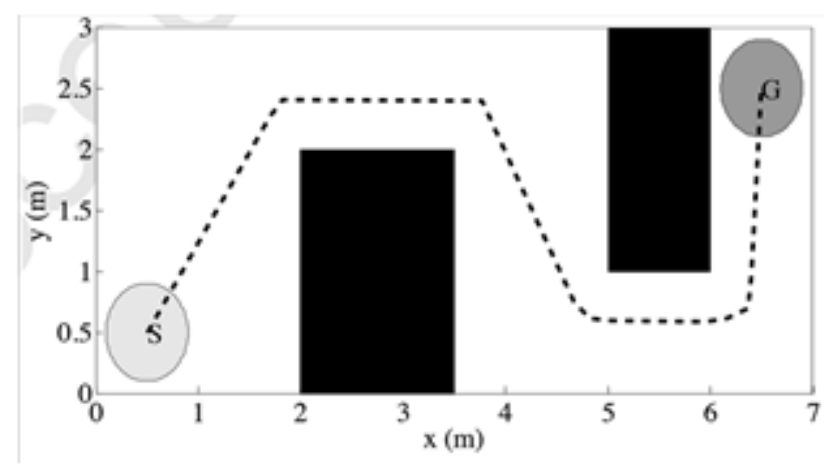

Fig. 7. Path trajectory selected by ABC-EP controller [24]

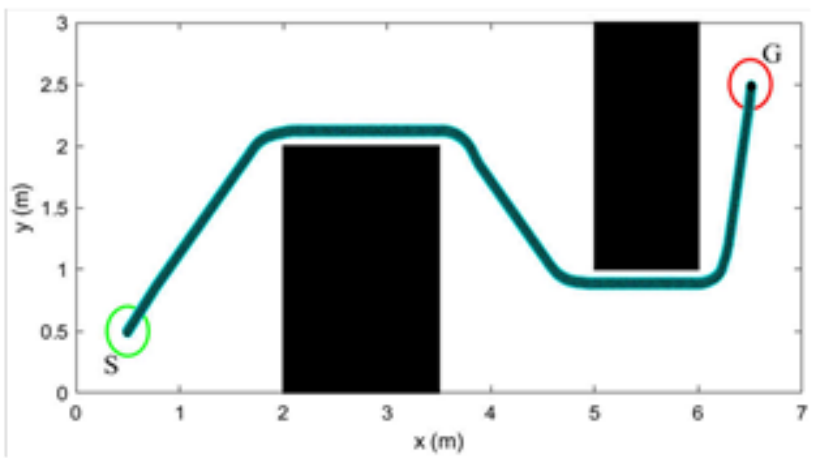

Fig. 8. Path trajectory selected by IMCIWO approach

TABLE III. COMPaRISON OF PATH LENGTH USING ABC-EP [24] CONTROLLER AND IMCIWO APPROACH

\begin{tabular}{|c|c|c|c|}
\hline Sl. No. & $\begin{array}{c}\text { ABC-EP } \\
\text { Controller }[24]\end{array}$ & $\begin{array}{c}\text { IMCIWO } \\
\text { Approach }\end{array}$ & $\begin{array}{c}\text { Improvement } \\
(\%)\end{array}$ \\
\hline 1. & 10.35 & 9.6 & 7.81 \\
\hline 2. & 10.35 & 10.1 & 2.48 \\
\hline 3. & 10.35 & 8.7 & 18.97 \\
\hline Avg. & $\mathbf{1 0 . 3 5}$ & $\mathbf{9 . 4 7}$ & $\mathbf{9 . 7 5}$ \\
\hline
\end{tabular}


Then the Bezier curve provides a smooth trajectory by utilizing the initial turning angle to provide my MCIWO approach. It is compared against IWO and MCIWO, where the proposed controller comes out as a clear winner. Additionally, it is analyzed in simulation and experimental terrains. The snapshots show that the robot reaches the target with no collision. The tabular comparison of simulation and experimental results in reference to path length and time spent shows an acceptable relation with deviation under $5 \%$. Ultimately, it has been compared with an existing technique to evaluate its acceptance of other developed controllers. Results show an improvement of $9.75 \%$. These results justify the selection of the IMCIWO approach as it shows acceptable robustness, efficiency, and acceptance in complicated workspaces. In the future, this can be utilized in for uneven terrain.

\section{REFERENCES}

[1] N. Bej, A. Pandey, A. K. Kashyap, and D. R. Parhi, "Optimum Navigation of Four-Wheeled Ground Robot in Stationary and Nonstationary Environments Using Wind-Driven Optimization Algorithm," in Innovative Product Design and Intelligent Manufacturing Systems, Springer, 2020, pp. 931-941.

[2] A. Pandey, A. K. Kashyap, D. R. Parhi, and B. K. Patle, “Autonomous mobile robot navigation between static and dynamic obstacles using multiple ANFIS architecture," World J. Eng., vol. 16, no. 2, pp. 275 286, Apr. 2019.

[3] A. K. Kashyap and A. Pandey, "Optimized Path Planning for ThreeWheeled Autonomous Robot Using Teaching-Learning-Based Optimization Technique," in Advances in Materials and Manufacturing Engineering, 2020, pp. 49-57.

[4] K. P. Lagaza, A. K. Kashyap, and A. Pandey, "Spider Monkey Optimization Algorithm Based Collision-Free Navigation and Path Optimization for a Mobile Robot in the Static Environment," in Advances in Mechanical Engineering, no. Ic, 2020, pp. 1459-1473.

[5] A. K. Kashyap, K. P. Lagaza, and A. Pandey, "Dynamic Path Planning for Autonomous Mobile Robot using Minimum Fuzzy Rule Based Controller with Avoidance of Moving Obstacles," in 2018 International Conference on Recent Innovations in Electrical, Electronics \& Communication Engineering (ICRIEECE), 2018, pp. 3330-3335.

[6] A. K. Kashyap and A. Pandey, "Different Nature-Inspired Techniques Applied for Motion Planning of Wheeled Robot: A Critical Review," Int. J. Adv. Robot. Autom., vol. 3, no. 2, pp. 1-10, Jul. 2018

[7] P. Karkowski, S. Oßwald, and M. Bennewitz, "Real-time footstep planning in 3D environments," in 2016 IEEE-RAS 16th International Conference on Humanoid Robots (Humanoids), 2016, pp. 69-74.

[8] J. Ido, Y. Shimizu, Y. Matsumoto, and T. Ogasawara, "Indoor navigation for a humanoid robot using a view sequence," Int. J. Rob. Res., vol. 28, no. 2, pp. 315-325, 2009.

[9] A. K. Kashyap, D. R. Parhi, and S. Kumar, "Dynamic Stabilization of NAO Humanoid Robot Based on Whole-Body Control with
Simulated Annealing," Int. J. Humanoid Robot., vol. 17, no. 03, p. 2050014, Jun. 2020.

[10] A. K. Kashyap, A. Pandey, A. Chhotray, and D. R. Parhi, "Controlled Gait Planning of Humanoid Robot NAO Based on 3D-LIPM Model," Available SSRN 3552498, 2020.

[11] Y. Zhong and B. Ai, "A modified ant colony optimization algorithm for multi-objective assembly line balancing," Soft Comput., vol. 21, no. 22, pp. 6881-6894, 2017.

[12] N. Habib, D. Purwanto, and A. Soeprijanto, "Mobile robot motion planning by point to point based on modified ant colony optimization and Voronoi diagram," in 2016 International Seminar on Intelligent Technology and Its Applications (ISITIA), 2016, pp. 613-618.

[13] A. K. Kashyap, D. R. Parhi, M. K. Muni, and K. K. Pandey, "A hybrid technique for path planning of humanoid robot NAO in static and dynamic terrains," Appl. Soft Comput., vol. 96, p. 106581, Nov. 2020.

[14] P. B. Kumar, C. Sahu, and D. R. Parhi, "A hybridized regressionadaptive ant colony optimization approach for navigation of humanoids in a cluttered environment," Appl. Soft Comput., vol. 68, pp. 565-585, Jul. 2018.

[15] P. B. Kumar, M. K. Muni, and D. R. Parhi, "Navigational analysis of multiple humanoids using a hybrid regression-fuzzy logic control approach in complex terrains," Appl. Soft Comput., vol. 89, p. 106088, Apr. 2020.

[16] A. K. Rath, D. R. Parhi, H. C. Das, M. K. Muni, and P. B. Kumar, "Analysis and use of fuzzy intelligent technique for navigation of humanoid robot in obstacle prone zone," Def. Technol., vol. 14, no. 6, pp. 677-682, 2018.

[17] C. Sahu, D. R. Parhi, and P. B. Kumar, "An approach to optimize the path of humanoids using adaptive ant colony optimization," J. Bionic Eng., vol. 15, no. 4, pp. 623-635, 2018.

[18] N. Razmjooy and M. Khalilpour, "A new design for PID controller by considering the operating points changes in Hydro-Turbine Connected to the equivalent network by using Invasive Weed Optimization (IWO) Algorithm," Int. J. Information, Secur. Syst. Manag., vol. 4, no. 2, pp. 468-475, 2015.

[19] S. Karimkashi and A. A. Kishk, "Invasive weed optimization and its features in electromagnetics," IEEE Trans. Antennas Propag., 2010.

[20] Y. R. Naidu and A. K. Ojha, "A hybrid version of invasive weed optimization with quadratic approximation," Soft Comput., 2015.

[21] O. Abedinia, A. Akbari Foroud, N. Amjady, and H. A. Shayanfar, "Modified invasive weed optimization based on fuzzy PSS in multimachine power system," in Proceedings of the 2012 International Conference on Artificial Intelligence, ICAI 2012, 2012.

[22] A. R. Mehrabian and C. Lucas, "A novel numerical optimization algorithm inspired from weed colonization," Ecol. Inform., 2006.

[23] F. Yamaguchi, Curves and surfaces in computer aided geometric design. Springer Science \& Business Media, 2012.

[24] M. A. Contreras-Cruz, V. Ayala-Ramirez, and U. H. HernandezBelmonte, "Mobile robot path planning using artificial bee colony and evolutionary programming," Appl. Soft Comput., vol. 30, pp. 319328, May 2015. 